\title{
Electrically Charged Vortex Solution in Born-Infeld Theory
}

\author{
E. Moreno ${ }^{a *}$, C. Núñez ${ }^{b \dagger}$ and F.A. Schaposnik $k^{b \ddagger}$ \\ ${ }^{a}$ Physics Department, City College of the City University of New York \\ New York NY 10031, USA \\ Physics Department, Baruch College, The City University of New York \\ New York NY 10010, USA \\ ${ }^{b}$ Departamento de Física, Universidad Nacional de La Plata \\ C.C. 67, 1900 La Plata, Argentina
}

(January 1, 2018)

\begin{abstract}
We obtain electrically charged vortex solutions for the Born-Infeld Higgs system with a Chern Simons term. We analyse numerically these solutions, comparing their properties with those of "normal" Nielsen-Olesen vortices and also show that no charged vortex solutions exist in BornInfeld theory when the Chern Simons term is absent.
\end{abstract}

PACS numbers: 11.27.+d 11.15.-q 11.10.-z

\section{INTRODUCTION}

Born-Infeld (BI) electrodynamics [1]- 2] has recently received much attention in connection with string theory and brane dynamics [3]- 国 (see [5] for a review and references). Classical configurations have been studied for different models in which the gauge field dynamics is governed by a BI Lagrangian and in this way domain wall, vortex and monopole solutions were constructed [6]- 8] and also gravitating solutions relevant in supergravity were found [8]- [9]. Supersymmetry and BPS saturated solutions in connection with D-brane dynamics have also been investigated [10][11.

An important point in the BI theory is that it admits finite energy static solutions (originally proposed in [1]- [2] to describe the electron) which are not source free like solitons but need pointlike sources which can be interpreted as the ends of electric-flux carrying strings. In particular, for some solutions discussed in [8] (called there BIons) strings look like tubes joining smoothly onto a p-brane.

The solutions we find in the present work are not BIonic but source free regular solitons of a BI theory. They are related to Nielsen-Olesen strings and in this sense they also look like tubes which are in fact electrically charged vortices (in contrast with those presented in [6] which carry magnetic flux but no electric charge).

Since in contrast with Maxwell electrodynamics BI theory allows for finite energy solutions which correspond to the field of electric charges, one could envisage to construct finite energy electrically charged vortex solutions just by studying a Born-Infeld-Higgs system, the non-linear electrodynamics version of the Nielsen-Olesen model [12] (which does not admit finite energy charged solutions). As we shall see, even allowing singularities as electric sources for the vortex charge, such solutions do not exist. Only if one adds a Chern-Simons (CS) term, finite energy BI charged vortices can be found, in analogy with what happens in the Maxwell case [13]- [17].

Of course, introducing a CS term implies that static, $z$-independent vortices in $3+1$ dimensions should be considered as static configurations in $2+1$. Hence, they should be thought more as planar configurations than as string-like objects but one should expect that their main properties should coincide with those arising when instead of a CS term (forcing to work in $2+1$ ) one couples fermions to the BI model [18]. It should be also noted that the resulting electric field is radial, in contrast with the configurations discussed in [10] in which the direction of the electric field defines the orientation of the string.

\footnotetext{
${ }^{*}$ Supported by CUNY Collaborative Incentive Grant 991999

${ }^{\dagger}$ CONICET

${ }^{\ddagger}$ Investigador CICBA, Argentina
} 
The plan of the paper is the following: in section II we discuss the Born-Infeld-Chern-Simons model coupled to a Higgs field. We show that charged vortex solutions exist in this model by solving numerically the Euler-Lagrange equations. The relevant properties of the solution are also discussed in this section. We then show in section III that, as it happens for Maxwell theory, Born-Infeld-Higgs theory (without CS term) does not admit finite energy regular solutions corresponding to magnetic vortices carrying electric charge. We give in section IV a brief summary of our results.

\section{THE BORN-INFELD-CHERN-SIMONS-HIGGS MODEL}

We shall consider the $2+1$ dimensional Lagrangian density for a complex scalar field $\phi$ minimally coupled to a $U(1)$ gauge field with dynamics governed by a Born-Infeld (BI) action [1]- [2] plus a Chern-Simons action

$$
L=-\beta^{2}\left(\sqrt{1+\frac{1}{2 \beta^{2}} F_{\mu \nu} F^{\mu \nu}}-1\right)+\frac{\kappa}{4 \pi} \varepsilon^{\mu \nu \alpha} A_{\mu} F_{\nu \alpha}+\frac{1}{2} D_{\mu} \phi^{*} D^{\mu} \phi-V[\phi]
$$

with

$$
\begin{gathered}
F_{\mu \nu}=\partial_{\mu} A_{\nu}-\partial_{\nu} A_{\mu} \\
D_{\mu} \phi=\left(\partial_{\mu}+i e A_{\mu}\right) \phi
\end{gathered}
$$

and $V[\phi]$ a symmetry breaking potential such that

$$
\left.\frac{\delta V[\phi]}{\delta \phi}\right|_{\phi=\eta}=0
$$

The constant $\beta$ has the dimensions of the electromagnetic field, $[\beta]=m^{3 / 2}$, while $[e]=m^{1 / 2}$ and $[\kappa]=m$. For fields much weaker than the critical field value $\beta$, Lagrangian (1) reduces to the Maxwell-Chern-Simons-Higgs Lagrangian.

It should be noted that models in which the kinetic term for the Higgs field appears together with the $F_{\mu \nu}^{2}$ in the square root can be considered. This possibility was discussed in [6] in connection with Bogomol'nyi equations for BI systems. We will discuss this alternative in section III.

The equations of motion resulting from (11) are

$$
\partial_{\mu}\left(\frac{F^{\mu \nu}}{\mathcal{R}}\right)+\frac{\kappa}{2 \pi} \varepsilon^{\mu \nu \alpha} F_{\nu \alpha}=j^{\nu}
$$

and

$$
\square \phi+2 i e A_{\mu} \partial^{\mu} \phi-e^{2} A_{\mu} A^{\mu} \phi=-\frac{\partial V}{\partial \phi^{*}}
$$

with

$$
\mathcal{R}=\sqrt{1+\frac{1}{2 \beta^{2}} F_{\mu \nu} F^{\mu \nu}}
$$

and

$$
j^{\nu}=\frac{i e}{2}\left(\phi^{*} \partial^{\nu} \phi-\phi \partial^{\nu} \phi^{*}\right)-e^{2}|\phi|^{2} A^{\nu}
$$

In Born-Infeld theories, the electric field $E^{i}$ and magnetic field $B^{i}$ are defined as usual as

$$
E^{i}=F^{0 i} \quad B^{i}=\frac{1}{2} \varepsilon^{i j k} F_{j k}
$$

while the electric induction $D^{i}$ and the magnetic intensity $H^{i}$, take the form

$$
D^{i}=f^{0 i} \quad H^{i}=\frac{1}{2} \varepsilon^{i j k} f_{j k}
$$


with

$$
f_{i j}=\frac{F_{i j}}{\mathcal{R}}
$$

In the limit $\beta^{2} \rightarrow \infty$ the BI action coincides with the Maxwell action so that Lagrangian (1) becomes the usual Maxwell-Chern-Simons-Higgs Lagrangian and $D^{i} \rightarrow E^{i}, H^{i} \rightarrow B^{i}$.

As it is well known, the Abelian Higgs model with the usual Maxwell action (and no Chern-Simons term) has finite energy axially symmetric static solutions, the well-honored Nielsen-Olesen vortices [12]. Vortex solutions carry quantized magnetic flux but, in contrast with monopoles [20], they are necessarily neutral since the existence of an electric charge implies infinite energy. Indeed, consider the $\beta^{2} \rightarrow \infty$ limit in which Lagrangian (1) becomes, for $\kappa=0$, the usual Abelian Higgs model Lagrangian with an electric charge $Q$ which can be defined as

$$
Q \equiv \int d^{2} x j^{0}=\int d^{2} x \partial_{i} E^{i}=\int_{S^{\infty}} d x_{i} E^{i}-\int_{S^{0}} d x_{i} E^{i}
$$

where $S^{\infty}$ and $S^{0}$ are the circles at $\rho \rightarrow \infty$ and $\rho \rightarrow 0$ respectively. Consistency of the coupled equations of motion imply that $E^{i} \rightarrow 0$ at infinity so one needs $|\vec{E}| \rightarrow 1 / \rho$ as $\rho \rightarrow 0$ in order to have non-zero electric charge. But this behavior implies that the vortex has infinite energy. The only way to evade this no-go result is to make dynamics governed by something else than the Maxwell action. In particular, one can add a Chern-Simons term endowing the vortex with charge through its well-known relation with magnetic flux forced by eqs. of motion [21]. This has been worked out in refs. [13]- 17] where Abelian and non-Abelian electrically charged vortices have been thoroughtfully investigated.

Another possibility is related to the Born-Infeld electromagnetism which allows singular electric induction $\vec{D}$ but with a regular electric field $\vec{E}$ and a finite energy. One may expect that regular charged vortex solutions could be constructed starting with a Born-Infeld Lagrangian instead of a Maxwell Lagrangian, even in the absence of ChernSimons terms. As we shall see, this is not the case and the Born-Infeld-Higgs system also requires a Chern-Simons term in order to exhibit finite energy solutions. We postpone this issue and first consider the complete theory, including the Chern-Simons action.

In polar coordinates the adequate axially symmetric ansatz reads

$$
\phi(\vec{x})=f(\rho) \exp (-i n \varphi) \quad A_{\varphi}(\vec{x})=-\frac{1}{\rho} A(\rho) \quad A_{0}(\vec{x})=A_{0}(\rho)
$$

We impose as boundary conditions

$$
\lim _{\rho \rightarrow \infty} f(\rho)=\eta \quad \lim _{\rho \rightarrow \infty} A(\rho)=-\frac{n}{e} \quad \lim _{\rho \rightarrow \infty} A_{0}(\rho)=0
$$

Concerning the origin

$$
f(0)=A(0)=0 \quad A_{0}(0)=c
$$

with $c$ a constant to be determined [19]. With this conditions one can easily see that the magnetic flux is quantized

$$
\Phi=\oint A_{i} d x^{i}=-\frac{2 \pi}{e} n
$$

Concerning the electric charge we use the formula (cf with (12))

$$
Q=\int d^{2} x j^{0}=\frac{\kappa}{2 \pi} \int d^{2} x \varepsilon^{i j} F_{i j}-\int d^{2} x \partial_{i} D^{i}=\frac{\kappa}{2 \pi} \Phi-\left.2 \pi \rho D_{\rho}\right|_{0} ^{\infty}
$$

As already recognized in 21], the presence of the Chern-Simons term forces the magnetic vortex to carry an electric charge. There is also in the present case, a second possible contribution since although the $D_{\rho}$ field has a long distance damping effected by the "photon" mass, one could in principle have a behavior at the origin of the form

$$
D_{\rho}(0) \sim-\frac{q}{2 \pi} \frac{1}{\rho}, \quad r \sim 0
$$

contributing to the electric charge, 


$$
Q=-\frac{\kappa}{e} n+q
$$

The compatibility of the imposed conditions at the origin and at infinity should be investigated through the analysis of the equations of motion (5)-(6). To this end, it will be convenient to define dimensionless quantities

$$
\begin{aligned}
& x(\tau)=n+e A(\tau) \\
& y(\tau)=A_{0}(\tau) / \eta \\
& z(\tau)=f(\tau) / \eta
\end{aligned}
$$

with

$$
\tau=e \eta \rho
$$

In terms of the new variables, eqs.(5)-(6) become

$$
\begin{gathered}
\tau \frac{d}{d \tau}\left(\frac{1}{\mathcal{R}} \frac{\dot{x}}{\tau}\right)-z^{2} x=\delta \dot{y} \tau \\
\frac{1}{\tau} \frac{d}{d \tau}\left(\frac{\tau}{\mathcal{R}} \dot{y}\right)-z^{2} y=\delta \frac{\dot{x}}{\tau} \\
\frac{1}{\tau} \frac{d}{d \tau}(\tau \dot{z})+z\left(y^{2}-\frac{x^{2}}{\tau^{2}}\right)-V^{\prime}(z)=0
\end{gathered}
$$

with

$$
\begin{gathered}
\mathcal{R}=\sqrt{1+\frac{1}{\bar{\beta}^{2}}\left(\frac{\dot{x}^{2}}{\tau^{2}}-\dot{y}^{2}\right)} \\
\bar{\beta}=\frac{\beta}{e \eta^{2}}, \quad \delta=\frac{\kappa}{\pi e \eta}, \quad V^{\prime}=\frac{1}{e^{2} \eta^{4}} \frac{\delta V}{\delta z}
\end{gathered}
$$

We have solved this equations numerically, by minimizing the action associated with Lagrangian (11) (Being the CS action metric independent, it does not enter explicitly in the expression for the energy). In fact, in terms of variables (20) the expressions for the action $S$ (omitting the time integral since we are looking for static solutions) and the energy $E$ for our model take the form

$$
\begin{gathered}
S=2 \pi \eta^{2} \bar{\beta}^{2} \int \tau d \tau\left(1-R+\frac{1}{2 \bar{\beta}^{2}}\left(-\frac{x^{2} z^{2}}{\tau^{2}}-\dot{z}^{2}+y^{2} z^{2}\right)+\delta \frac{\dot{x}}{\tau} y-\frac{1}{\bar{\beta}^{2}} \frac{\lambda}{8 e^{2}}\left(z^{2}-1\right)^{2}\right) \\
E=\int d^{2} x T_{00}=2 \pi \eta^{2} \bar{\beta}^{2} \int \tau d \tau\left(\frac{1}{R}\left(1+\frac{1}{\bar{\beta}^{2}} \frac{\dot{x}^{2}}{\tau^{2}}\right)-1+\frac{1}{2 \bar{\beta}^{2}}\left(\dot{z}^{2}+y^{2} z^{2}+z^{2} \frac{x^{2}}{\tau^{2}}\right)+\frac{1}{\bar{\beta}^{2}} \frac{\lambda}{8 e^{2}}\left(z^{2}-1\right)^{2}\right)
\end{gathered}
$$

Here $T_{\mu \nu}$ is the energy momentum tensor and we have used for the potential the usual form

$$
V[\phi]=\frac{\lambda}{8}\left(|\phi|^{2}-\eta^{2}\right)^{2}
$$

As noted above, the CS coefficient $\delta$ enters in the action but not in the energy of the vortex.

We shall now analyse the vortex equations (21)-(23). For simplicity we will concentrate in the case $n=1$. As a first step, let us study the asymptotic behavior of the solutions. If one assumes that the boundary conditions at infinity, eq.(14), are reached exponentially, the differential equations for large $\tau$ can be linearized around $x, y$ and $1-z$. It is then straightforward to deduce the asymptotic behavior of the solutions, which is independent of the value of $\beta$ :

$$
\begin{aligned}
& x(\tau)=c_{1} \tau K_{1}(\alpha \tau) \\
& y(\tau)=c_{2} K_{0}(\alpha \tau) \\
& z(\tau)=1-c_{3} K_{0}(\sqrt{\lambda} \tau)
\end{aligned}
$$


Here either

$$
c_{1}=c_{2} \quad \text { and } \quad \alpha=\frac{\delta+\sqrt{\delta^{2}+4}}{2}
$$

or

$$
c_{1}=-c_{2} \quad \text { and } \quad \alpha=\frac{-\delta+\sqrt{\delta^{2}+4}}{2} .
$$

It can be proved that for $\delta>0$ only the solution with $c_{1}=c_{2}$ exists while for $\delta<0$ only the solution with $c_{1}=-c_{2}$ exists 15.

To obtain a detailed profile of the vortex solution we solved numerically the differential equations using a relaxation method for boundary values problem [23]. Such method determines the solution by starting with a guess and improving it iteratively. For the case under consideration, the initial guess

$$
\begin{aligned}
& x(\tau)=1-\left(1-e^{-a \tau}\right)^{2} \\
& y(\tau)=c\left(1-\left(1-e^{-b \tau}\right)^{2}\right) \\
& z(\tau)=1-e^{-d \tau}
\end{aligned}
$$

perfectly works with any reasonable election of the parameters $a, b, c$ and $d$. By minimizing the action with the ansatz (33), (with $\beta=1, \delta=0$ and $\lambda=1$ ) we choosed the values $a=0.67, b=0.78, c=-0.03, d=0.86$. The profile of the vortex solutions for different values of $\beta$, for $\delta=0.1$ and $\lambda=1$ are shown in figs. 1.2. Note that the solution we have found implies that the behavior given in $(18)$ is to be excluded so that the vortex electric charge is related to its magnetic flux in the usual form dictated by the CS term, $Q=-(\kappa / e) n$.

It can be seen from the numerical solutions that, as expected, for large values of $\beta$ the solution differs very little from the Maxwell-Chern-Simons-Higgs vortices [13] [15]. However as $\beta$ decreases, the vortex profile changes notably and a remarkable peculiarity takes place: as $\beta$ approcahes to some critical value $\beta_{c}$, the magnetic field at the origin tends to infinity, and the numerical solution ceases to exist if $\beta$ is smaller than $\beta_{c}$ (see fig. 3) This actual value of $\beta_{c}$ depends on the remaining parameters $(\delta, \lambda)$ and its existence becomes more evident if one represents the vortex energy versus $\beta$ (for fixed values of $\delta$ and $\lambda$, see fig. 3). One can see in particular that the derivative of the energy with respect to $\beta$ diverges as $\beta$ approaches $\beta_{c}$. Even for $\delta=0$ (absence of Chern simons term) the $\beta_{c}$ is nonzero (and in fact takes its maximun value). Values of $\beta_{c}$ for differents values of $\delta$ are: $\delta=0.465$ for $\beta_{c}=0, \delta=0.448$ for $\beta_{c}=0.1, \delta=0.416$ for $\beta_{c}=0.2$, and $\delta=0.247$ for $\beta_{c}=1$.

Though we were unable to find an analytical argument to account for the existence of this singularity, we have enough numerical evidence that supports our claim. In fact, in addition to the singular behavior of the energy around $\beta_{c}$, and the absence of numerical solutions with $\beta<\beta_{c}$ using relaxation methods, we found a similar pattern when solving the equations by other methods (shooting algorithm for boundary value problem, etc.)

\section{NON-EXISTENCE OF CHARGED SOLUTIONS IN THE ABSENCE OF A CS TERM}

Let us first consider, instead of (1), a Born-Infeld-Higgs Lagrangian without the Chern-Simons term but including a $1 / \beta^{4}$ term coupling between the field strength and the Higgs field (appart from entering the Higgs field kinetic term in the BI square root)

$$
L=\beta^{2}\left(\sqrt{1-\frac{1}{2 \beta^{2}} F_{\mu \nu} F^{\mu \nu}-\frac{1}{\beta^{2}} D^{\mu} \phi^{*} D_{\mu} \phi+\frac{1}{\beta^{4}} \tilde{F}_{\mu} \tilde{F}_{\nu} D^{\mu} \phi^{*} D^{\nu} \phi-\frac{1}{2 \beta^{4}} D_{\mu} \phi * D^{\mu} \phi F_{\alpha \beta} F^{\alpha \beta}}-1\right)-V[\phi]
$$

This Lagrangian is the $2+1$ Abelian version of the one exhibiting dyonic solutions, investigated in [7]. In this last work it was shown that breaking of duality invariance $F_{\mu \nu} \rightarrow \tilde{F}_{\mu \nu}$ was necessary in order to have electrically charged monopoles, the coupling of the dual field $\tilde{F}_{\mu \nu}$ to the Higgs field doing this work. In the present $2+1$

dimensional case, one has to couple the scalar to the dual $\tilde{F}_{\mu}=\frac{1}{2} \varepsilon_{\mu \nu \alpha} F^{\nu \alpha}$ and the simplest way is that given in (34). Moreover, inclusion of the Higgs field kinetic energy term in the Born-Infeld square root was necessary in order to have Bogomol'nyi equations. An ansatz of the form (13) together with the notation (20) leads to the following equations of motion for the gauge field components

$$
\tau \frac{d}{d \tau}\left[\frac{1}{\mathcal{R}} \frac{\dot{x}}{\tau}\left(-1+\frac{1}{\bar{\beta}^{2}}\left(\dot{z}^{2}+\frac{x^{2}}{\tau^{2}} z^{2}\right)\right)-\frac{1}{\mathcal{R}} \frac{1}{\bar{\beta}^{2}} z^{2} \dot{y} y \frac{x}{\tau}\right]=\frac{z^{2}}{\mathcal{R}}\left[x-\frac{1}{\bar{\beta}^{2}}\left(x \dot{y}^{2}+\dot{y} \dot{x} y-x\left(\frac{\dot{x}^{2}}{\tau^{2}}-\dot{y}^{2}\right)\right)\right]
$$




$$
\frac{1}{\tau} \frac{d}{d \tau}\left[\frac{\tau}{\mathcal{R}} \dot{y}\left(1+\frac{1}{\bar{\beta}^{2}}\left(y^{2} z^{2}-\dot{z}^{2}-\frac{x^{2}}{\tau^{2}} z^{2}\right)\right)-\frac{1}{\mathcal{R}} \frac{1}{\bar{\beta}^{2}}\left(\dot{x} y z^{2} \frac{x}{\tau}+\dot{y} \frac{x^{2}}{\tau} z^{2}\right)\right]=\frac{z^{2}}{\mathcal{R}}\left(-y+\frac{1}{\bar{\beta}^{2}}\left(y \dot{y}^{2}-\dot{y} \dot{x} \frac{x}{\tau^{2}}\right)\right.
$$

We now assume a vortex-like behavior near the origin of the form

$$
\begin{gathered}
x=1+\frac{1}{2} d_{1} \tau^{2} \\
y=c+y_{1} \tau+\frac{1}{3} y_{3} \tau^{3} \\
z=a_{0} \tau+\frac{1}{3} a_{1} \tau^{3}
\end{gathered}
$$

The behavior (37) corresponds to a vortex with $n=1$ units of magnetic flux. This is consistent with the Higgs field having a simple zero at the origin as implied by (39). The resulting magnetic and electric field at the origin have the following behavior:

$$
\begin{gathered}
\frac{\dot{x}}{\tau}=d_{1} \\
\dot{y}=y_{1}+y_{3} \tau^{2}
\end{gathered}
$$

Now, eqs.(35)-(36) impose certain relations among the coefficients in expansions (37)-(39). Indeed, compatibility implies:

$$
\begin{gathered}
a_{0}^{2}=\frac{\bar{\beta}^{2}}{2} \\
c=0
\end{gathered}
$$

One should also note also that the behavior (18) implies

$$
\frac{\dot{y}}{R} \sim \frac{q}{2 \pi \eta} \frac{1}{\tau} \quad \text { for } \quad \tau \sim 0
$$

so that from $(41)$ we see that one necessarily has $\mathcal{R} \sim \tau$. Now, one can easily see that formulae (42)-(43) gives for $\mathcal{R}$ a behavior of the form

$$
\mathcal{R} \sim \frac{a_{0}}{\bar{\beta}^{2}} \quad \text { for } \quad \tau \sim 0
$$

this showing that a charged vortex solution with finite energy does not exist in the theory with dynamics determined by Lagrangian (34). One can convince onself that other possible $\beta^{4}$ terms do not change this situation.

\section{SUMMARY}

We have studied the classical equations of motion of a Born-Infeld Higgs model looking for (magnetic) vortex solutions carrying also electric charge (the analogous of dyon solutions in the case of monopoles). These kind of solutions do not exist in Maxwell-Higgs model: electric charge implies in this case inifinite energy, as originally signaled in ref. 20]. Since precisely the problem of infinite energy of charged solutions in pure electromagnetism was overcome by Born and Infeld with their proposal, one could expect that finite energy charged vortex could exist. We have proven however in section III that this is not the case.

On the contrary, finite energy charged vortices do exist when a Chern-Simons term is added to the Maxwell Lagrangian. We have proven that this also happens for the Born-Infeld Lagrangian. We have seen numerically that for $\beta$ (the Born-Infeld parameter) sufficiently large, the corresponding Born-Infeld solution differs very little from the Maxwell-Chern-Simons-Higgs vortices. When $\beta$ decreases the vortex profile changes notably and there exists a critical 
value of $\beta$ where the magnetic field $H$ at the origin and $\partial E / \partial \beta$ ( $E$ being the energy of the vortex solution) diverges. There is no solution for $\beta$ less then $\beta_{c}$. We have not been able to find an analytical argument explaining this behavior which we show is not a byproduct of our numerical procedure.

We conclude that vortex solutions in Born Infeld theories present many interesting features which deserve a thoroughfull study. In particular, Bogomol'nyi equations should be analysed, not only for their per se interest but also in connection with supersymmetric extensions and brane dynamics. We hope to report on these issues in a forthcoming work.

Acknowledgements: F.A.S. is partially suported by CICBA, Argentina and a Commission of the European Communities contract No:C11*-CT93-0315. This work is supported in part by funds provided by the U.S. Department of Energy (D.O.E.) under cooperative research agreement \# DF-FC02-94ER40818.

[1] M. Born, Proc. R. Soc. London A143 (1934) 410.

[2] M. Born and M. Infeld, Proc. R. Soc. London A144 (1934) 425.

[3] E.S. Fradkin and A.A.Tseytlin, Phys. Lett. 163B (1985) 123; A.A. Tseytlin, Nucl. Phys. B276 (1986) 391.

[4] R.G. Leigh, Mod. Phys. Lett. A4 (1989) 2767 and 2073.

[5] J.Polchinski, Tasi Lectures on D-Branes, hep-th/9611050.

[6] A. Nakamura and K. Shiraishi, Int. Jour. of Mod. Phys. A6 (1991) 2635.

[7] A. Nakamura and K. Shiraishi, Hadronic Jour. 14 (1991) 369.

[8] G. Gibbons, hep-th/9709027.

[9] G. Gibbons and D.A. Rasheed, Nucl.Phys. B454 (1995) 185.

[10] C.G. Callan and J.M. Maldacena, hep-th/9708147.

[11] J.P. Gauntlett, J. Gomis and P.K. Townsend, hep-th/9711205.

[12] H.B. Nielsen and P. Olesen Nucl. Phys. B61 (1973) 45.

[13] K. Paul and A. Khare Phys. Lett. B 174 (1986) 420.

[14] H. de Vega and F.A. Schaposnik, Phys. Rev. Lett. 56 (1986) 2564. Phys. Rev. D34 (1986) 3206.

[15] G. Lozano, M.V. Manías and F.A. Schaposnik, Phys. Rev. D38 (1988) 601.

[16] J. Hong, Y. Kim and P.-Y. Pac, Phys. Rev. Lett. 64 (1990) 2230.

[17] R. Jackiw and E. Weinberg, Phys. Rev. Lett. 64 (1990) 2234.

[18] R. Jackiw and P. Rossi, Nucl. Phys. B190[FS3] (1981) 681.

[19] L. Cugliandolo, G. Lozano, M.V. Manías and F.A. Schaposnik, Mod .Phys. Lett. A6 (1991) 479.

[20] B. Julia and A. Zee, Phys. Rev. D11 (1975) 2227.

[21] S. Deser, R. Jackiw and S. Templeton, Ann. of Phys. (N.Y.) 140 (1982) 372.

[22] H. de Vega and F.A. Schaposnik, Phys. Rev. D14 (1976) 1100.

[23] W.H. Press, S.A. Teukolsky and W.T. Vetterling, Numerical Recipes: The art of Scientific Computing, Cambridge University Press, (1992). 


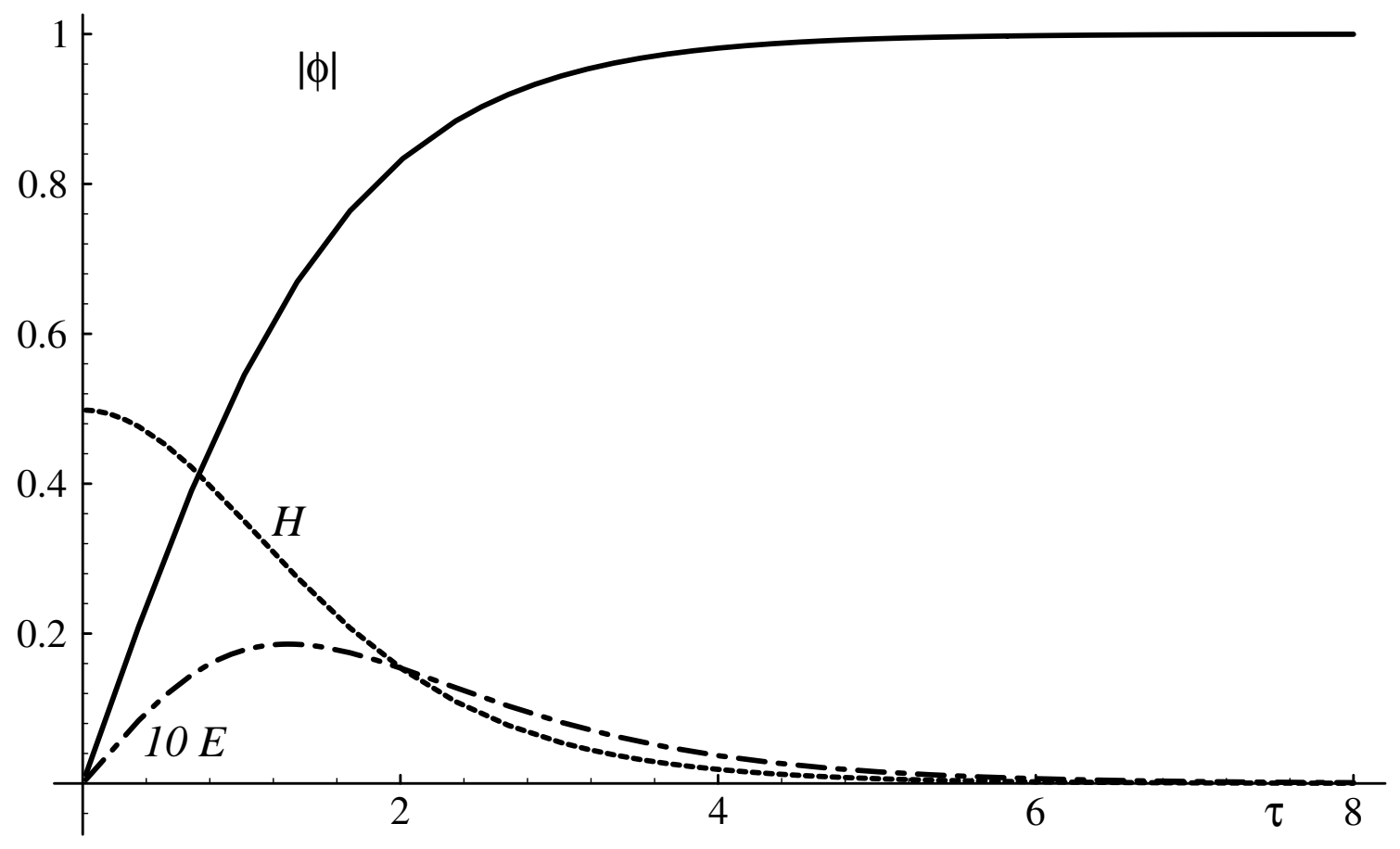

FIG. 1. Vortex solution for $\beta=10, \delta=0.1$ and $\lambda=1$. The full line corresponds to the modulus of the Higgs field, $|\phi(\tau)|$, the dashed line correspond to the magnetic field $H(\tau)$ and the dot-dashed line to the electric field $E_{r}(\tau)$. 


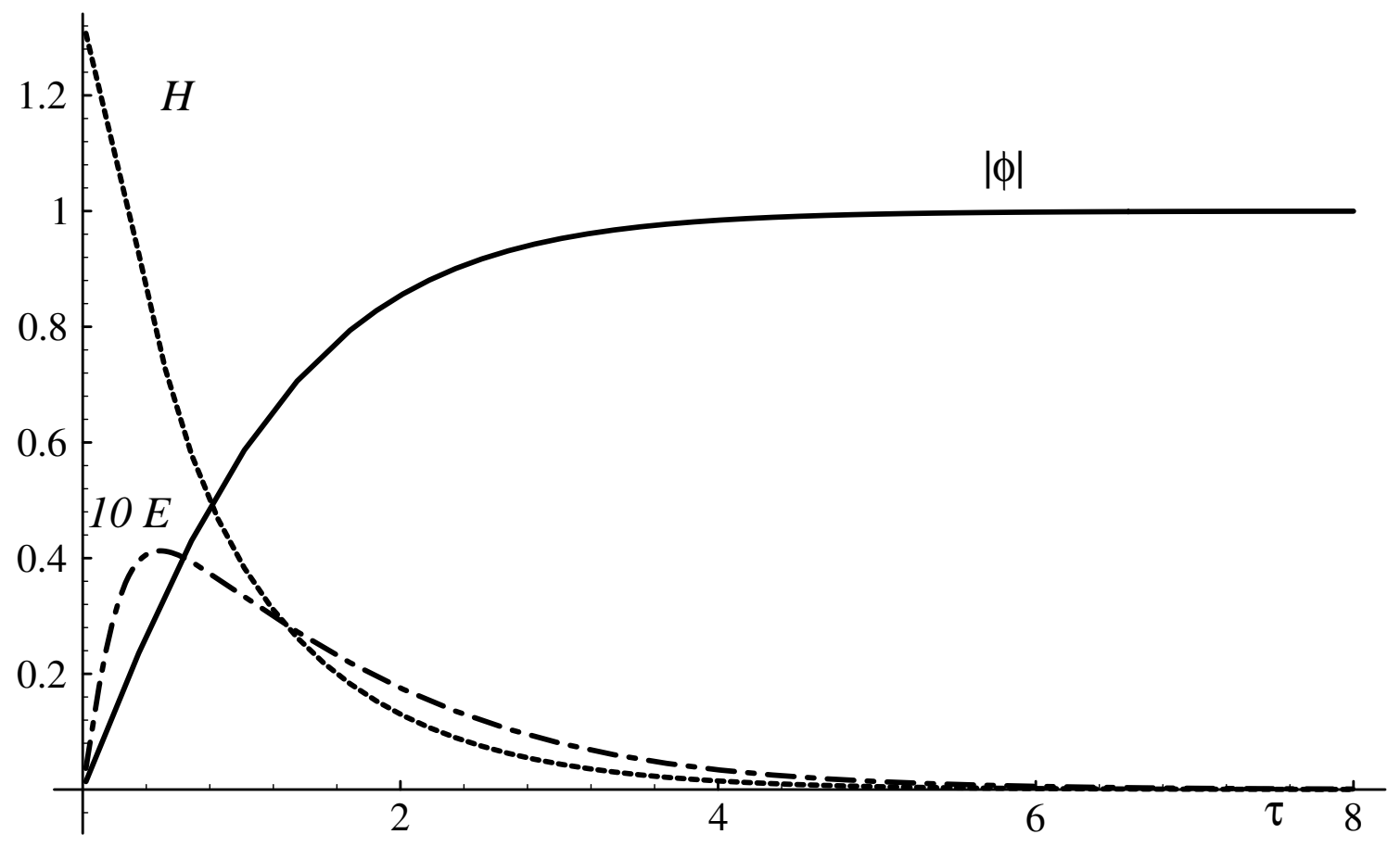

FIG. 2. Vortex solution for $\beta=0.5, \delta=0.1$ and $\lambda=1$. The full line corresponds to the modulus of the Higgs field, $|\phi(\tau)|$, the dashed line correspond to the magnetic field $H(\tau)$ and the dot-dashed line to the electric field $E_{r}(\tau)$. 


\section{Energy}

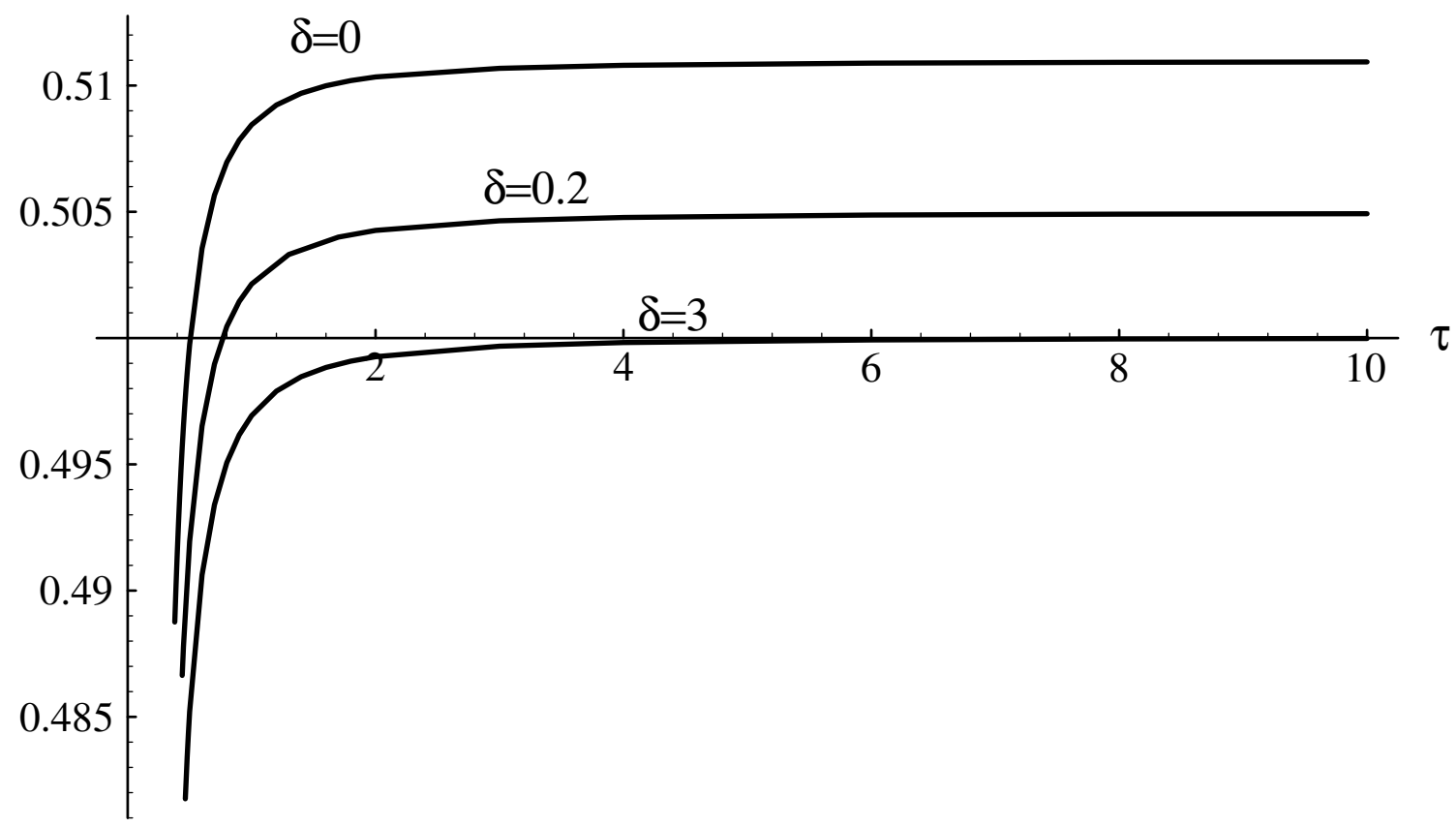

FIG. 3. Energy of the vortex as a function of $\beta$ for different values of $\delta$ ( and $\lambda=1$ ). The first line from the top corresponds to $\delta=0$ (non-charged vortex), the second line corresponds to $\delta=0.2$ and the last one to $\delta=3.0$. 